\title{
Évaluation de quelques méthodes de calcul des pieux forés
}

\author{
Assessment of some design methods for bored piles
}

\author{
M. BUSTAMANTE, R. FRANK \\ Laboratoire Central des Ponts et Chaussées* \\ S. CHRISTOULAS \\ Université Technique Nationale d'Athènes*"
}

Rev. Franç. Géotech. n 54, pp. $39-52$ (janvier 1991)

\section{Résumé}

On traite, tout d'abord, du problème de la capacité portante des pieux forés, en confrontant cinq différentes méthodes de calcul à partir d'essais en place, d'origine française et étrangère, aux résultats de 20 essais de chargement de pieux en vraie grandeur. On aborde également le problème du calcul du tassement des pieux isolés et l'on propose des corrélations entre le module pressiométrique ou la résistance au cône et le module du sol, pour l'application de la méthode élastique de POULOS et DAVIS. Enfin, la méthode par fonctions de transfert de charge (courbes de mobilisation du frottement latéral $t-z$ ) de FRANK et ZHAO est également appliquée et le logiciel correspondant PIVER est présenté.

\begin{abstract}
This paper first deals with the problem of the bearing capacity of bored piles by comparing five different design methods based on in-situ testing, of French or foreign origin, to the results of 20 full scale loading tests on piles. The settlement of isolated piles is also dealt with and correlations between the pressuremeter modulus or the cone resistance and the soil modulus is proposed for application of the elastic method of POULOS and DAVIS. Finally, the load transfer method (side friction mobilization curves $t-z$ ) of FRANK and ZHAO is also applied and the corresponding computer program PIVER is presented.
\end{abstract}

- 58, boulevard Lefebure, 75732 Paris Cedex 15.

* Polytechnic Campus Zografos, 15700 Athènes, Grèce. 


\section{INTRODUCTION}

L'essai de chargement est le moyen le plus efficace pour dimensionner les fondations sur pieux. Son intérêt est encore accru si l'on a pu mettre en place une instrumentation, le long du fût du pieu d'essai, permettant la mesure séparée des frottements latéraux et de l'effort de pointe en fonction des déplacements du pieu.

L'interprétation des résultats peut, soit servir à la justification, a posteriori, du prédimensionnement effectué par une méthode de prévision usuelle, soit constituer un moyen propre de calcul pour la fondation en question.

Par ailleurs, l'exploitation systématique d'essais de pieux existants permet, bien évidemment, une évaluation précise des méthodes de calcul disponibles ou encore la mise au point de méthodes nouvelles. Les règles françaises récentes de calcul de la capacité portante des pieux à partir d'essais pressiométriques ou pénétrométriques, basées sur l'exploitation de nombreux essais de chargement en vrai grandeur (BUSTAMANTE et GIANESELLI, 1981 ; LCPC et SETRA, 1985) en est une excellente illustration.

Le dimensionnement dune fondation sur pieux nécessite ;

- l'estimation de la capacité portante (axiale) des pieux isolés ;

- l'estimation du tassement des pieux isolés sous charge de service

- l'estimation des déplacements et des efforts sous charges de flexion;

- l'estimation d'éventuels effets de groupe.

La présente étude a pour but, d'une part l'évaluation et la comparaison de certaines méthodes étrangères de calcul de la capacité portante (CGS, 1985 ; DIN 4014, 1977) moins familières aux lecteurs francophones que les méthodes de calcul pressiométriques ou pénétrométriques utilisées en France et, d'autre part l'établissement de corrélations nouvelles entre le module pressiométrique ou la résistance au cône et le module d'Young (ou « module du sol ») pour les pieux forés, afin de pouvoir utiliser la méthode élastique, bien connue, de calcul des tassements selon POULOS et DAVIS (1980). Par ailleurs, certains résultats de calcul des tassements par la méthode pressiométrique de FRANK et ZHAO (1982) sont rappelés.

Ces interprétations sont basées sur les résultats de 20 essais de pieux forés instrumentés, réalisés par les Laboratoires des Ponts et Chaussées, ces dernières années. Ils font partie de la banque de données d'essais de pieux du Laboratoire Central, comprenant à l'heure actuelle 279 essais sur 80 sites différents et 151 pieux, dont 126 étaient instrumentés tout le long du fût. Ces 20 essais ont été choisis au hasard, en cherchant uniquement à couvrir différents types de sols et modes d'exécution de pieux forés.

\section{PRÉSENTATION DES MÉTHODES DE CALCUL}

\subsection{Capacité portante des pieux isolés}

Les méthodes de calcul de la capacité portante des pieux peuvent être classées en trois catégories:

- les méthodes " classiques "à partir des essais de laboratoire (caractéristiques de résistance au cisaillement). Ces méthodes sont décrites dans la plupart des cours de mécanique des sols. On peut, notamment, consulter CHRISTOULAS (1988) ;

- les méthodes dynamiques, que ce soit tant les formules classiques dites de "battage " que les méthodes, plus récentes, utilisant l'analyse de la propagation des ondes (voir BOURGES et FRANK, 1989, par exemple) ;

- les méthodes utilisant directement les résultats d'essais en place: essentiellement, SPT (Standard Penetration Test), CPT (Essai de Pénétration au Cône ou Essai de Pénétration Statique) et PMT (Essai au Pressiomètre Ménard). La description de ces essais peut être trouvée dans ISSMFE-TC 16 (1989) pour le SPT et le CPT et dans AFNOR (1990) pour le PMT.

Le but de la présente étude est d'évaluer:

- la méthode SPT du Canadian Foundation Engineering Manual (CGS, 1985 ; MEYERHOF, 1976), ( méthode 1 ) ;

- les 3 méthodes CPT suivantes :

- du Canadian Foundation Engineering Manual (CGS, 1985 ; « méthode 2 ») ;

- de la norme allemande DIN 4014 (1977), ( méthode $3 »)$;

- et du Document technique unifié français DTU $n^{\circ} 13.2$ (1983) (BUSTAMANTE et GIANESELLI, 1981), (" méthode 4 ») :

- et, enfin, la méthode pressiométrique PMT recommandée par le LCPC et le SETRA (1985), suite aux travaux de BUSTAMANTE et GIANESELLI (1981), (« méthode 5 »),

\subsubsection{Méthode 1 (SPT, CGS, 1985)}

Pour les sols pulvérulents, la charge limite $Q_{1}$ d'un pieu isolé est, d'après le Canadian Foundation Engineering Manual :

$$
\mathrm{Q}_{1}=\mathrm{mNA}_{\mathrm{b}}+\mathrm{n} \overline{\mathrm{N}} \mathrm{PD} \text { en } \mathrm{kN}
$$

avec :

$\mathrm{m}$ : coefficient empirique ;

$m=120$ pour les pieux forés $(m=400$ pour les pieux battus);

$\mathrm{N}$ : le nombre de coups de lessai SPT (pour l'enfoncement de $30 \mathrm{~cm}$ ) au niveau de la pointe du pieu ;

$A_{b}$ : laire de la section droite de la pointe du pieu, en $\mathrm{m}^{2}$;

$\mathrm{n}$ : coefficient empirique, $\mathrm{n}=1$ pour les pieux forés ( $n=2$ pour les pieux battus);

$\overline{\mathrm{N}}$ : le nombre moyen de coups de l'essai SPT le long du fût du pieu ;

D : la fiche du pieu, en $\mathrm{m}$;

$\mathrm{P}$ : le périmètre du pieu, en $\mathrm{m}$. 


\subsubsection{Méthode 2 (CPT, CGS, 1985)}

Pour les limons et sables lâches à denses, le Canadian Foundation Engineering Manual propose de calculer la charge limite d'un pieu isolé à partir de résultats de pénétration statique (CPT) de la manière suivante :

$$
Q_{1}=q_{c} A_{b}+f_{s} P D
$$

avec :

$\mathrm{q}_{\mathrm{c}}$ : résistance au cône (ou résistance de pointe statique) en pointe du pieu, mesurée au CPT :

$\mathrm{f}_{\mathrm{s}}$ : frottement latéral local unitaire moyen mesuré au CPT (on recommande l'utilisation d'un cône équipé d'un manchon de frottement).

Notons qu'on recommande, pour les pieux de diamètre $\mathrm{B}$ supérieur à $500 \mathrm{~mm}$, de prendre en compte une valeur de $\mathrm{q}_{c}$ inférieure à la valeur moyenne mesurée, ou même égale à la plus petite valeur mesurée.

Dans la présente étude, ne disposant pas de mesures au manchon de frottement, les corrélations suivantes entre le frottement latéral unitaire local $f_{s}$ et la résistance au cône q sont utilisées (d'après SANGLERAT, cité par CASSAN (1988), ou notre expérience propre) :

- sables denses et graviers: $\quad f_{\mathrm{s}}=\mathrm{q}_{\mathrm{c}} / 150$;

- sables moyens: $\quad \mathrm{f}_{\mathrm{s}}=\mathrm{q}_{\mathrm{c}} / 100$;

- limons et sols intermédiaires: $f_{s}=q_{c} / 60$;

- argiles : $\quad f_{s}=q_{c} / 50$;

- craies: $\quad f_{s}=q_{s} / 100 ;$

- calcaires: $\quad \mathrm{f}_{\mathrm{s}}=\mathrm{q}_{\mathrm{c}} / 150$.

\subsubsection{Méthode 3 (CPT, DIN 4014, 1977)}

La norme DIN 4014 Part 2 (1977) s'applique aux pieux forés de grand diamètre (supérieur à $0,5 \mathrm{~m}$ le long du fût et à $1 \mathrm{~m}$ en pointe).

La pression de pointe $\mathrm{g}(\mathrm{s})$ est donnée en fonction du tassement $\mathrm{s}$, respectivement au tableau 1 pour les sols non cohérents et au tableau 2 pour les sols cohérents.

Le frottement latéral limite unitaire $\mathrm{q}_{\mathrm{s}}$ est donné en fonction de la résistance au cône $q_{c}$ et de la profondeur pour les sols non cohérents (tableau 3), et en fonction de la cohésion non drainée $c_{u}$ pour les sols cohérents (tableau 4). On suppose que $\mathrm{q}_{\mathrm{s}}$ est mobilisé pour un tassement de $2 \mathrm{~cm}$ pour les sols non cohérents et de $1 \mathrm{~cm}$ pour les sols cohérents.

Les tableaux 1 à 4 permettent donc de construire toute la courbe charge-tassement du pieu. La charge limite $Q_{1}$ est obtenue par:

$$
Q_{1}=q_{p} A_{b}+\sum_{i} q_{s i} p_{i} D_{i}
$$

avec :

$\mathrm{q}_{\mathrm{p}}=\mathrm{q}\left(\mathrm{s}_{\mathrm{i}}\right)$ : résistance de pointe (charge unitaire limite) où : $s_{1}=15 \mathrm{~cm}$ pour les sols non cohérents, $\mathrm{s}_{1}=0,05 \mathrm{~B}_{\mathrm{b}}$ pour les sols cohérents, $\mathrm{B}_{\mathrm{b}}$ étant le diamètre de la pointe du pieu ;
Tableau 1. - Pression de pointe en fonction du tassement en sol non cohérent

de résistance moyenne (résistance au cône $q_{c}$ comprise entre 10 et $15 \mathrm{MPa}$ (DIN 4014, 1977)

Table 1. - Pile base resistance depending on the settlements in non cohesive soil of average strength

(penetrometer end bearing pressure $q_{s}$ equal to 10 up to $15 \mathrm{MPa}$

\begin{tabular}{|c|c|}
\hline \multicolumn{2}{|c|}{ Pieux sans base élargie } \\
\hline $\begin{array}{c}\text { Tassement } \\
\text { s } \\
\mathrm{cm}\end{array}$ & $\begin{array}{c}\text { Pression de pointe* } \\
\mathrm{q}(\mathrm{s}) \\
\mathrm{MPa}\end{array}$ \\
\hline 1 & 0,5 \\
2 & 0,8 \\
3 & 1,1 \\
$15^{* *}$ & 3,4 \\
\hline \multicolumn{2}{|c|}{ Pieux avec base élargie } \\
\hline 1 & 0,35 \\
2 & 0,65 \\
3 & 0,9 \\
$15^{*}$ & 2,4 \\
\hline
\end{tabular}

- Valeurs intermédiaires a obtenir par interpolation linéaire.

- Tassement supposé pour la charge limite $Q_{1}$.

Tableau 2. - Pression de pointe en fonction du tassement en sol cohérent moyennement consistant $\left(U_{c} \simeq 1\right)$ (DIN 4014, 1977)

Table 2. - Pile base resistance depending on the settlements in cohesive roughly semi-firm soil (coefficient of consistency $I_{c}=1$ )

\begin{tabular}{|c|c|}
\hline $\begin{array}{c}\text { Tassement } \\
\mathrm{s} \\
\mathrm{cm}\end{array}$ & $\begin{array}{c}\text { Pression de pointe* } \\
\mathrm{q}(\mathrm{s}) \\
\mathrm{MPa}\end{array}$ \\
\hline $0,2 \cdot \mathrm{s}_{1}$ & 0,5 \\
$0,3 \cdot \mathrm{s}_{1}$ & 0,7 \\
$\mathrm{~s}_{1}=0,05 \mathrm{~B}_{\mathrm{b}} * *$ & 1,2 \\
\hline
\end{tabular}

- Valeurs intermédiaires à obtenir par interpolation linésire.

* s tassement supposé pour la charge limite $\mathrm{Q}_{1}$.

$\mathrm{B}_{\mathrm{b}}=$ diamètre de la pointe du pieu.

Tableau 3. - Frottement latéral limite unitaire en sol non cohérent (DIN 4014, 1977)

Table 3. - Skin friction values in non-cohesive soil

\begin{tabular}{|l|c|c|c|}
\hline $\begin{array}{c}\text { Résistance } \\
\text { du sol } \\
\text { non cohérent }\end{array}$ & $\begin{array}{c}\text { Résistance } \\
\text { au cône } \\
\mathrm{q}_{\mathrm{c}} \mathrm{MPa}\end{array}$ & $\begin{array}{c}\text { Profondeur } \\
\mathrm{m}\end{array}$ & $\begin{array}{c}\text { Frottement } \\
\text { latéral } \\
\mathrm{q}_{\mathrm{s}} \mathrm{MPa}\end{array}$ \\
\hline Très faible & $<5$ & - & 0 \\
\hline Faible & 5 à 10 & $\begin{array}{r}0 \text { à } 2 \\
2 \text { à } 5 \\
>5\end{array}$ & $\begin{array}{l}0 \\
0,03 \\
0,05\end{array}$ \\
\hline Moyenne & 10 à 15 & $\begin{array}{c}0 \text { à } 2 \\
2 \text { à } 7,5 \\
>7,5\end{array}$ & $\begin{array}{l}0 \\
0,045 \\
0,075\end{array}$ \\
\hline Elevée & $>15$ & $\begin{array}{c}0 \text { à } 2 \\
2 \text { à } 10 \\
>10\end{array}$ & $\begin{array}{l}0 \\
0,06 \\
0,1\end{array}$ \\
\hline
\end{tabular}


Tableau 4. - Frottement latéral limite unitaire en sol cohérent

Table 4. - Skin friction values in cohesive soil

\begin{tabular}{|c|c|}
\hline $\begin{array}{c}\text { Cohésion non drainée } c_{u} \\
\text { du sol cohérent } \mathrm{MPa}\end{array}$ & $\begin{array}{c}\text { Frottement latéral } \\
\mathrm{q}_{\mathrm{s}} \mathrm{MPa}\end{array}$ \\
\hline 0 & 0 \\
0,025 & 0,025 \\
0,1 & 0,04 \\
0,2 & 0,05 \\
\hline
\end{tabular}

$p_{1}$ et $D_{j}$, périmètre du pieu dans la couche de sol i et épaisseur de cette couche, de frottement latéral limite unitaire $\mathrm{q}_{\mathrm{si}}$.

Quand les hypothèses sur la qualité des sols indiquées aux tableaux 1 et 2 ne sont pas satisfaites, la charge peut être augmentée ou doit être réduite suivant le cas, sur la base de résultats d'essais de chargement ou sur la base de données obtenues pour des pieux comparables dans des conditions de sols comparables.

\subsubsection{Méthode 4}

\section{(CPT, BUSTAMANTE et GIANESELLI, 1981)}

La méthode de BUSTAMANTE et GIANESELLI (1981), reprise par le DTU no 13.2 (1983), utilise uniquement la résistance au cône $\mathrm{q}_{\mathrm{c}}$ mesurée au CPT. L'expression de la charge limite est la suivante :

$$
Q_{1}=k_{c} q_{c} A_{b}+\sum_{i} q_{s i} p_{i} D_{i}
$$

avec :

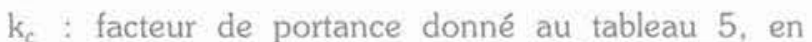
fonction de la nature du sol, de la résistance au cône $\mathrm{q}_{c}$ et de la catégorie du pieu :

$\mathrm{q}_{\mathrm{s}}$ : le frottement latéral unitaire limite. On prend, en fait :

$$
\mathrm{q}_{\mathrm{s}}=\min \left(\mathrm{q}_{\mathrm{c}} / \alpha ; \mathrm{q}_{\mathrm{s}, \max }\right) \text {. }
$$

les valeurs du coefficient $\alpha$ et de $q_{s, \max }$ étant données au tableau 5 , en fonction de la nature du sol, de la résistance au cône $\mathrm{q}_{\mathrm{c}}$ de la catégorie de pieu et de la nature du fût.

\subsubsection{Méthode 5 (PMT, LCPC et SETRA, 1985)}

La méthode pressiométrique recommandée par le LCPC et le SETRA est largement inspirée des travaux de BUSTAMANTE et GIANESELLI (1981), mais ne lui est pas strictement équivalente. La charge limite d'un pieu isolé est calculée de la manière suivante:

$$
\mathrm{Q}_{1}=\left[\mathrm{k}_{\mathrm{p}}\left(\mathrm{p}_{\mathrm{le}}-\mathrm{p}_{\mathrm{o}}\right)+\mathrm{q}_{\mathrm{o}}\right] \mathrm{A}_{\mathrm{b}}+\sum_{i} \mathrm{q}_{\mathrm{s} !} \mathrm{p}_{i} \mathrm{D}_{1}
$$

avec :

$k_{\mathrm{p}}$ : facteur de portance, donné en fonction de la nature du sol et de la catégorie de pieu au tableau 6 ;

$\mathrm{P}_{\mathrm{l}}$ : pression limite équivalente en pointe du pieu (à partir des mesures au pressiomètre entre les niveaux $D$-a et $D+3 a$, où $a=\max (B / 2 ; 0,5$ m)) ;

$\mathrm{p}_{\mathrm{o}}$ : contrainte horizontale totale au niveau de la pointe au moment de l'essai pressiométrique;

Tableau 5. - Méthode pénétrométrique. Valeurs du facteur de portance $k_{c}$ et du coefficient $\alpha$

\begin{tabular}{|c|c|c|c|c|c|c|c|c|c|c|c|c|c|}
\hline \multirow{3}{*}{ Nature du sol } & \multirow{3}{*}{$\left(\begin{array}{c}9_{c} \\
\left(10^{5} \mathrm{~Pa}\right)\end{array}\right)$} & \multirow{2}{*}{\multicolumn{2}{|c|}{$\begin{array}{l}\text { Facteur de portance } \\
\qquad k_{c}\end{array}$}} & \multicolumn{4}{|c|}{ Coefficient $\alpha$} & \multicolumn{6}{|c|}{ Valeur maximale de $q_{\mathrm{s}}\left(10^{5} \mathrm{~Pa}\right)(\%)$} \\
\hline & & & & \multicolumn{2}{|c|}{ Pieu foré } & \multicolumn{2}{|c|}{ Pieu battu } & \multicolumn{2}{|c|}{ Pieu foré } & \multicolumn{2}{|c|}{ Pieu battu } & \multicolumn{2}{|c|}{ Pieu injecté } \\
\hline & & $\begin{array}{l}\text { Pieu foré } \\
\text { (1) }\end{array}$ & $\begin{array}{l}\text { Pieu battu } \\
\text { (2) }\end{array}$ & $\begin{array}{l}\text { Fut } \\
\text { béton }\end{array}$ & Tubé & $\begin{array}{l}\text { Fût } \\
\text { béton }\end{array}$ & $\begin{array}{l}\text { Fût } \\
\text { métal }\end{array}$ & $\begin{array}{l}\text { Fût } \\
\text { béton }\end{array}$ & Tubé & $\begin{array}{l}\text { Fôt } \\
\text { báton }\end{array}$ & $\begin{array}{l}\text { Fôt } \\
\text { métal }\end{array}$ & $\begin{array}{c}\text { Faible } \\
\text { pression }\end{array}$ & $\begin{array}{l}\text { Hausse } \\
\text { pression }\end{array}$ \\
\hline Argile molle et vase & $<10$ & 0,4 & 0,5 & 30 & 30 & 30 & 30 & 0,15 & 0,15 & 0,15 & 0,35 & 0,35 & - \\
\hline $\begin{array}{l}\text { Argile moyennement } \\
\text { compacte }\end{array}$ & $\begin{array}{c}10 \text { à } \\
50\end{array}$ & 0,35 & 0,45 & 40 & 80 & 40 & 80 & $\begin{array}{l}(0.8) \\
0.35\end{array}$ & $\begin{array}{c}10,8) \\
0,35\end{array}$ & $\begin{array}{l}10,8) \\
0,35\end{array}$ & 0,35 & 0.8 & $\geqslant 1,2$ \\
\hline Limon et sable lâche & $\leqslant 50$ & 0,4 & 0,5 & 60 & 150 & 60 & 120 & 0,35 & 0,35 & 0,35 & 0,35 & 0,8 & - \\
\hline $\begin{array}{l}\text { Argile compacte } \\
\text { a faide et limon } \\
\text { compact }\end{array}$ & $>50$ & 0,45 & 0,55 & 60 & 120 & 60 & 120 & $\begin{array}{l}10,8) \\
0,35\end{array}$ & $\begin{array}{c}(0,8) \\
0,35\end{array}$ & $\begin{array}{l}10,81 \\
0.35\end{array}$ & 0,35 & 0,8 & $\geqslant 2,0$ \\
\hline $\begin{array}{l}\text { Sable et grave } \\
\text { moyennement compacts }\end{array}$ & $\begin{array}{ll} & 50 \\
\text { à } & 120\end{array}$ & 0,4 & 0,5 & 100 & 200 & 100 & 200 & $\begin{array}{c}(1,2) \\
0,8\end{array}$ & $\begin{array}{l}10,8) \\
0,35\end{array}$ & $\begin{array}{c}(1,2) \\
0,8\end{array}$ & 0,8 & 1,2 & $\geqslant 2,0$ \\
\hline $\begin{array}{l}\text { Craie altérée } \\
\text { à fragmentée }\end{array}$ & $>50$ & 0.2 & 0,4 & 60 & 80 & 60 & 80 & $\begin{array}{c}(1,5) \\
1,2\end{array}$ & $\begin{array}{c}(1,2) \\
0.8\end{array}$ & $\begin{array}{c}(1,5) \\
1,2\end{array}$ & 1,2 & 1,5 & $\geqslant 2,0$ \\
\hline $\begin{array}{l}\text { Sable et grave compacts } \\
\text { à très compacts }\end{array}$ & $>120$ & 0.3 & 0,4 & 150 & 300 & 150 & 200 & $\frac{(1,5)}{1,2}$ & $\begin{array}{c}(1,2) \\
0,8\end{array}$ & $\begin{array}{l}(1,5) \\
1,20\end{array}$ & 1,20 & 1,5 & $\geqslant 2.0$ \\
\hline
\end{tabular}
(BUSTAMANTE et GIANESELLI, 1981)

Table 5. - Penetrometer method Values of bearing factor $k_{c}$ and coefficient $\alpha$

(1) $\mathrm{Y}$ compris l'ensemble des fondations relevant du premier groupe (1). Voir $\$$ utilisation des abaques définissant les valeurs de $k$.

(2) $\mathrm{Y}$ compris l'ensemble des fondations relevant du deuxième groupe (II), Voir $\S$ utilisation des abaques définissant les valeurs de $k$.

(*) Les valeurs entre pararenthèses correspondent pour les pieux forés, à une exécution soignée du pieu et une technologie de mise en ceuvré susceptible de remanier au minimum le sol au contact du fût. Pour les pieux battus, par contre, à un resserrement du sol sur le pieu après battage. 
Tableau 6. - Méthode pressiométrique. Valeurs du facteur de portance $k_{p}$ (SETRA et LCPC, 1985) Table 6. - Pressuremeter Method. Values of bearing factor $k_{p}$

\begin{tabular}{|l|c|c|}
\hline & Sans refoulement du sol & Avec refoulement du sol \\
\hline Argiles-Limons & 1,2 & 1,8 \\
Sables-Graves & $1,1(1)$ & 3,2 à $4,2(2)$ \\
Craie-Marnes - Marno-Calcaire & 1,8 & 2,6 \\
Rocher altéré ou fragmenté & 1,1 à $1,8(3)$ & 1,8 à $3,2(3)$ \\
\hline
\end{tabular}

(1) Le peu de résultats dont on dispose pour les graves, et pour les graves compactes en particulier, laisse penser que les valeurs proposées peuvent être conservatrices. Celles-ci pourront être redéfinies ultérieurement. Rappelons toutefois qu'il importe que les valeurs de $\mathrm{p} 1$ prises en compte correspondent aux valeurs réalles de ce paramètre.

(2) Les résultats expêrimentaux disponibles pour cette catégorie de pieux dans les sables mettent en évidence une variation du facteur de portance en pointe avec la pression limite. Cette variation traduirait le resserrage du sol autour du pieu, qui apparait d'autant plus marqué que le sol est lâche. Ainsi, on adoptera pour $\mathrm{k}_{\mathrm{g}}$ la valeur de 4,2 , pour $1<1,0 \mathrm{MPa}$ et 3,2 pour $\mathrm{P}_{1}>3 \mathrm{MPa}$, en interpolant linéairement pour les valeurs intermédiaires de la pression fimite. Pour les graves sableuses et les graves propres, on ne dispose pas de données expérimentales suffisantes. Dans l'attente de telles données, on pourra adopter les mêmes valeurs que pour les sables.

(3) On ne dispose que de peu de données expérimentales en ce qui concerne le comportement en pointe des pieux dans le rocher aitéré ou frag. menté. Pour les cas connus, la charge limite des pieux n'a pu être atteinte, compte tenu notamment des valeurs élevées de pressions limites qui caractérisent ces formations. Aussi, s'il est reconnu que le matériau concerné s'apparente assez étroitement à l'un des sols meubles pour lesquels le tableau propose une valeur particulière de $k_{p}$, on adoptera celle-ci (par exemple $k_{p}=1,1$ pour un pieu foré dans un rocher aitéré ou fragmenté susceptible d'avoir un comporteemnt proche d'un sable ou d'une grave compacte). Une telle valeur de $k$ p probablement assez prudente, permettra toutefois, dans de nombreux cas, de justifier le dimensionnement par référence à la résistnce du matériau constitutif du pieu, compte tenu des valeurs êlevées de la pression limite de ces formations. En tout état de cause, des valeurs de $k_{p}$ supérieures à celles indiquées ne pourront être généralement adoptées que sur justification expérimentale.

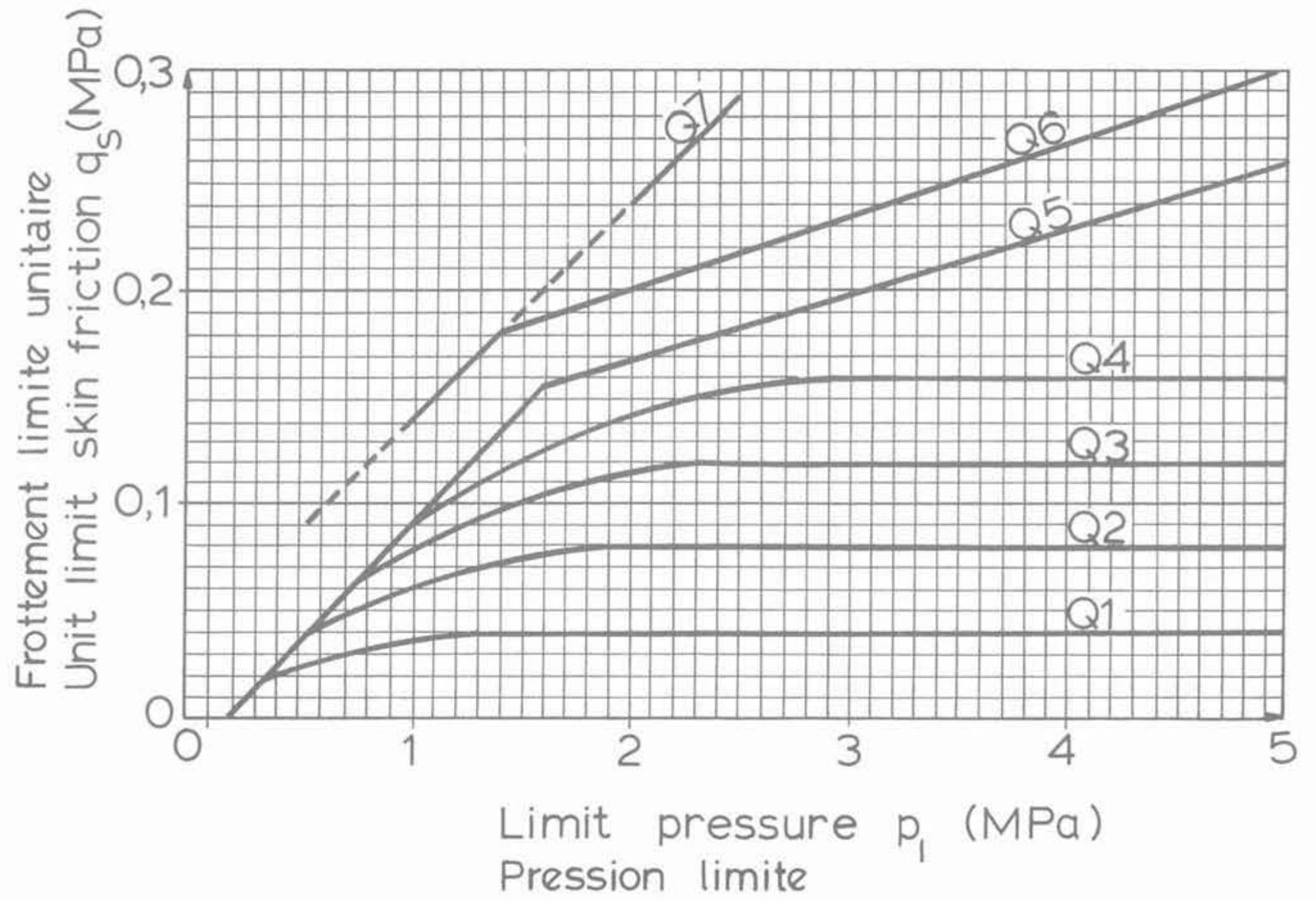

Fig. 1. - Méthode pressiométrique. Frottement latéral unitaire limite. (Pour le choix de la courbe, voir tableau 7) (LCPC, SETRA, 1985).

Fig. 1. - Pressuremeter method. Limit unit skin friction. (For the choice of the curve, see table 7) 
Tableau 7. - Méthode pressiométrique. Choix des abaques pour le calcul du frottement latéral unitaire limite $q_{s}$ (SETRA et LCPC, 1985)

Table 7. - Pressuremeter method. Choice of charts for the determination of limit unit skin friction $q_{s}$

\begin{tabular}{|c|c|c|c|c|c|c|}
\hline & $\begin{array}{l}\text { Argiles } \\
\text { limons }\end{array}$ & Sables & Graves & Craie & $\begin{array}{c}\text { Marnes } \\
\text { Marno-calcaire }\end{array}$ & $\begin{array}{l}\text { Rocher altéré } \\
\text { ou fragmenté }\end{array}$ \\
\hline Foré simple & $\begin{array}{l}\mathrm{Q}_{1}{ }^{+} \\
\mathrm{Q} 2(2) \\
\text { (2) }\end{array}$ & & & $\begin{array}{l}\mathrm{O3}^{+} \\
\mathrm{O} 6^{+}(2)\end{array}$ & $\begin{array}{l}\mathrm{Q} 4^{+} \\
\mathrm{Q} 5\end{array}$ & $\mathrm{Q6}^{+}$ \\
\hline Foré boue & $\begin{array}{l}\mathrm{O} 1^{+} \\
\mathrm{O} 2(2)\end{array}$ & $\begin{array}{l}Q 1^{+}(6) \\
02\end{array}$ & $\begin{array}{l}\mathrm{O} 2(6) \\
\mathrm{O} 3\end{array}$ & $\begin{array}{l}\mathrm{O}^{+} \\
\mathrm{O} 6^{+}\end{array}$ & $\begin{array}{l}\mathrm{O} 4^{+} \\
\mathrm{O} 5\end{array}$ & $\mathrm{Q6}^{+}$ \\
\hline $\begin{array}{l}\text { Foré Tubé } \\
\text { tubage récupéré) }\end{array}$ & $\begin{array}{l}\mathrm{Q} 1^{+} \\
\mathrm{O} 2(4)\end{array}$ & $\begin{array}{l}\mathrm{Q}_{1}^{+}+(6) \\
02\end{array}$ & $\mathrm{Q}_{2}(6)$ & $\begin{array}{l}\mathrm{Q}^{+} \\
\mathrm{Q} 4^{+}\end{array}$ & Q4 & \\
\hline $\begin{array}{l}\text { Foré tubé } \\
\text { (tubage perdu) }\end{array}$ & Q1 & Q1 & $\mathrm{Q} 2$ & $\mathrm{Q} 2$ & $\mathrm{O}^{+}$ & \\
\hline Puits (1) & $\begin{array}{l}\text { Q2 } \\
\text { Q3 (5) }\end{array}$ & & & $04^{+}$ & 05 & $06^{+}$ \\
\hline $\begin{array}{l}\text { Métal battu } \\
\text { (tube fermé) }\end{array}$ & $\begin{array}{l}\text { Q1 }{ }^{+} \\
\text {Q2 (5) }\end{array}$ & Q2 & Q3 & Q4 & Q4 & $\mathrm{Q} 4^{+}(7)$ \\
\hline $\begin{array}{l}\text { Battu préfabriqué } \\
\text { (fât béton) }\end{array}$ & Q2 & Q3 & Q3 & $04^{+}$ & $\mathrm{Q} 4^{+}$ & $\mathrm{Q} 4^{+}(7)$ \\
\hline Battu moulé & Q2 & $\mathrm{Q}_{2}{ }^{+}$ & Q3 & Q4 & Q4 & \\
\hline Battu enrobé & $\mathrm{O} 2$ & $\mathrm{O3}^{+}$ & Q4 & $05^{+}$ & $\mathrm{Q}_{4}^{+}$ & \\
\hline Injecté basse pression & $\mathrm{O} 2^{+}$ & $03^{+}$ & $\mathrm{O} 3^{+}$ & $05^{+}$ & $\mathrm{O} 5^{+}$ & $06^{+}$ \\
\hline $\begin{array}{l}\text { Injecté } \\
\text { haute pression (8) }\end{array}$ & $05^{+}$ & $05^{+}$ & Q6 $6^{+}$ & $06^{+}$ & Q6 + & $\mathrm{Q} 7^{+}(9)$ \\
\hline
\end{tabular}

(1) Sans tubage ni virole foncés perdus (parois rugueuses).

(2) Réalésage et rainurage en fin de forage.

(3) Rèalésage et rainurage en fin de forage, réservé aux argiles raides $(p) \geqslant 1.5 \mathrm{MPa}$ ).

(4) Forage à sec, tube non louvoyé.

(5) Argiles raides $\left(p_{i} \geqslant 1,5 \mathrm{MPa}\right)$.

(6) Pieux de grande longueur (supérieure à $30 \mathrm{~m}$ )

(7) Si battage possible.

(8) Injection sélective et répétitive à faible débit.

(9) (8) et traitement préalable des massifs fissurés ou fracturés avec obturation des cavités. Concerne surtout les micropieux pour lesquels il est recommandé généralement de procéder à des essais de chargement si le nombre le justifie.

$q_{0}$ : contrainte verticale totale au niveau de la pointe après travaux :

$\mathrm{q}_{\mathrm{s}}$ : frottement latéral limite unitaire, fonction de la nature du sol, de la pression limite mesurée au pressiomètre, ainsi que du type exact de pieu (fig. 1 et tableau 7).

\subsection{Tassement des pieux isolés}

Deux méthodes de calcul sont habituellement utilisées pour estimer les tassements des pieux isolés sous charge de service;

- la méthode élastique de POULOS et DAVIS (1980), où le sol est modélisé par un continuum élastique linéaire isotrope ( $\mathrm{E}_{\mathrm{s}}$, module d'Young et $\nu$, coefficient de Poisson) ;

- la méthode des fonctions de transfert de charge ou méthode des « courbes $\mathrm{t}-\mathrm{z}$ ». L'interaction entre le sol et le pieu y est modélisée par des ressorts axiaux linéaires ou non, représentant les courbes de mobilisation du frottement latéral $\mathrm{t}(\mathrm{s})$ et de la pression de pointe $\mathrm{q}(\mathrm{s})$ en fonction du tassement au niveau correspondant. FRANK et ZHAO (1982) ont proposé des règles de construction de ces courbes en fonction du module pressiométrique $\mathrm{E}_{\mathrm{m}}$. Pour ce qui est de la résolution numérique, le programme PIVER est utilisé.

\subsubsection{Méthode élastique de POULOS et DAVIS, 1980}

La méthode de POULOS et DAVIS (1980) est basée sur la théorie de l'élasticité linéaire et isotrope et l'utilisation des formules de MINDLIN. On obtient le tassement du pieu en fonction des caractéristiques du pieu (fiche $\mathrm{D}$, diamètre $\mathrm{B}$ et module d'Young $\mathrm{E}_{\mathrm{p}}$ ), du module du sol $E_{\mathrm{s}}$ et de coefficients correcteurs représentant respectivement l'influence de la rigidité relative pieu-sol, l'influence du substratum et l'influence du coefficient de Poisson du sol. 
Plus précisément, le tassement s en tête du pieu s'exprime par :

$$
\mathrm{s}=\mathrm{Ql} / \mathrm{E}_{\mathrm{s}} \mathrm{B}
$$

avec :

$\mathrm{Q}$ : la charge pour laquelle on calcule le tassement du pieu:

I ; le coefficient d'influence :

- pour un pieu flottant,

$$
\mathrm{I}=\mathrm{I}_{\mathrm{o}} \mathrm{R}_{\mathrm{k}} \mathrm{R}_{\mathrm{h}} \mathrm{R}_{\nu}
$$

où :

$1_{0}$ est le coefficient d'influence pour un pieu incompressible, dans un milieu semi-infini et pour $\nu=$ 0.5 .

$\mathrm{R}_{\mathrm{k}}$ est le coefficient correcteur pour la compressibilité du pieu ;

$\mathrm{R}_{\mathrm{h}}$ est le coefficient correcteur pour la profondeur du substratum ;

$\mathrm{R}_{y}$ est le coefficient correcteur pour le coefficient de Poisson ;

- pour un pieu résistant en pointe,

$$
\mathrm{I}=\mathrm{I}_{\mathrm{o}} \mathrm{R}_{\mathrm{k}} \mathrm{R}_{\mathrm{b}} \mathrm{R}_{\boldsymbol{y}}
$$

où :

$I_{0}, R_{k}$ et $R_{y}$ sont les mêmes que pour le pieu flottant ;

$\mathrm{R}_{\mathrm{b}}$ est le coefficient correcteur pour la rigidité du substratum.

Tous les coefficients sont donnés, par POULOS et DAVIS (1980), sous forme d'abaques et permettent donc un calcul manue!. Signalons, par ailleurs, que la méthode a également été étendue au calcul du tassement des groupes de pieux.

La principale difficulté réside dans la connaissance du module du sol $E_{s}$. Le moyen le plus efficace pour déterminer $E_{5}$ est évidemment de recourir à un essai de chargement en vraie grandeur et d'analyser à l'envers les résultats obtenus, à l'aide de la méthode. Mais comme l'essai de chargement est une opération délicate et onéreuse (et, de plus, qui ne peut pas être envisagée pour un simple prédimensionnement), les auteurs ont proposé une corrélation entre le module du sol et, d'une part la cohésion non drainée $c_{u}$ pour les argiles, d'autre part l'indice de densité $\mathrm{I}_{\mathrm{D}}$ (densité relative) pour les sables. La figure 2 reproduit la corrélation proposée par POULOS et DAVIS (1980) pour les argiles, qui a été obtenue en analysant, à l'envers, un certain nombre d'essais de pieux en vraie grandeur.

CHRISTOULAS et PACHAKIS (1987) et CHRIS. TOULAS (1988) ont analysé, de la même manière, des essais de chargement de pieux battus moulés en Grèce, pour estimer des modules du sol équivalents. Des corrélations sont ainsi proposées avec le nombre de coups $N$ du SPT (fig. 3) et la résistance au cône $\mathrm{q}_{\mathrm{c}}$ mesurée avec le CPT (fig. 4).

\subsubsection{Méthode t-z (FRANK et ZHAO, 1982)}

Les premières propositions pour déterminer les courbes de mobilisation du frottement latéral et de l'effort de pointe à partir des résultats de l'essai pressiomé-

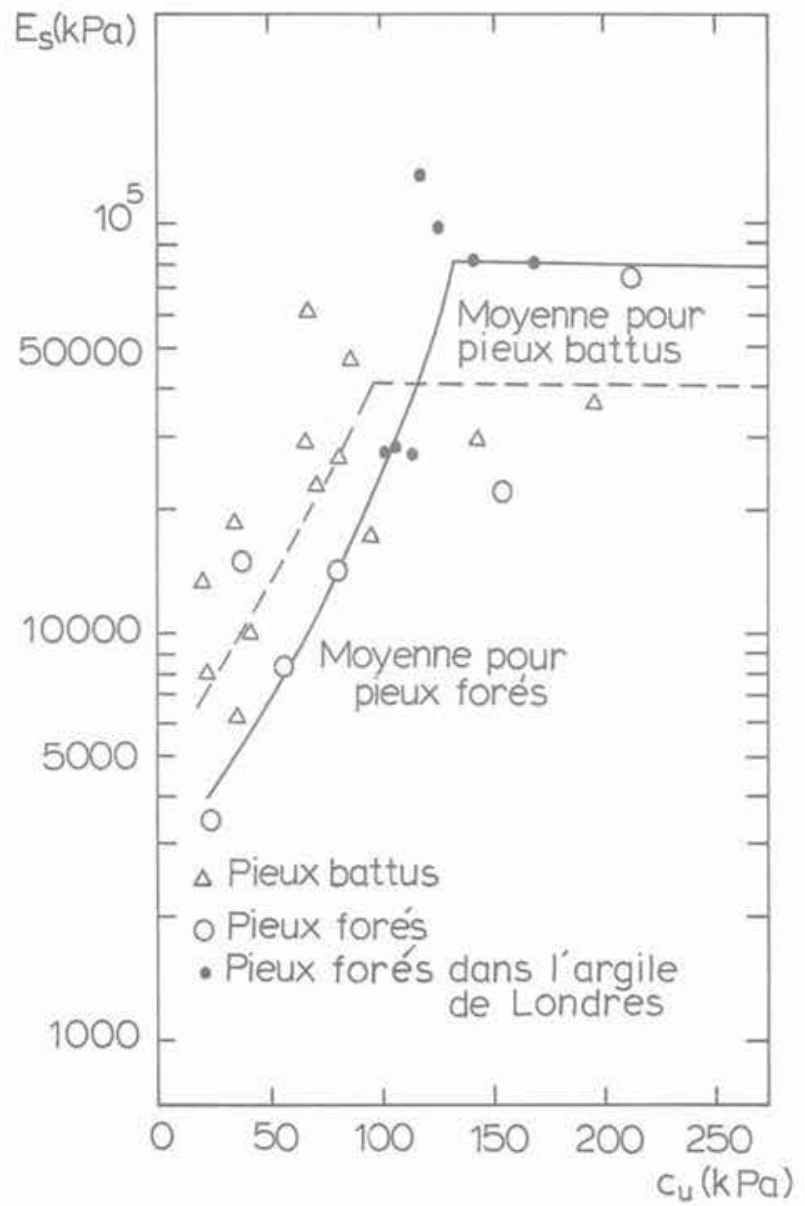

Fig. 2. - Corrélation entre le module du sol $E_{s}$ et la cohésion non drainée $c_{u}$ (POULOS et DAVIS, 1980).

Fig. 2. - Correlation between soil modulus $E_{s}$ and undrained shear strength $\mathrm{c}_{\mathrm{u}}$

trique sont dues à GAMBIN (1963) et CASSAN (1966, 1968). Les lois de mobilisation proposées par FRANK et ZHAO (1982), initialement pour les pieux forés dans les sols fins, sont représentées à la figure 5 .

Les paramètres de mobilisation $B$ et $R$ sont déterminés, pour tous types de pieux et de sols, à partir du module pressiométrique $\mathrm{E}_{\mathrm{M}}$, de la manière suivante:

- sols fins et roches tendres: $\quad B=E_{M} / r_{0}$

$$
\text { et } \quad \mathrm{R}=5,5 \mathrm{E}_{\mathrm{M}} / \mathrm{r}_{\mathrm{o}}
$$

$$
\text { - sols granulaires : } \begin{array}{rl}
\mathrm{B} & =0,4 \mathrm{E}_{\mathrm{M}} / \mathrm{r}_{\mathrm{o}} \\
\mathrm{R} & =2,4 \mathrm{E} \\
\mathrm{M} & \mathrm{r} / \mathrm{r}_{\mathrm{o}}
\end{array}
$$

où : $r_{0}$ est le rayon du pieu.

BUSTAMANTE, FRANK et GIANESELLI (1989) ont analysé, à l'aide de ces lois, 33 pieux forés, battus et injectés, fichés dans des argiles, craies, marnes et marno-calcaires et ont conclu que la méthode était très satisfaisante dans la plupart des cas. Dans les cas de tubes battus dans les sables et de pieux forés dans les marnes argileuses notamment, elle nécessite encore 
Pieux résistants en pointe < sans élargissement de la base Pieux flottants $<$ sans élargissement de la base +

Tous les pieux

$E_{s}=6,95 \mathrm{~N}-86,7(\mathrm{MPa}), r=0,91$

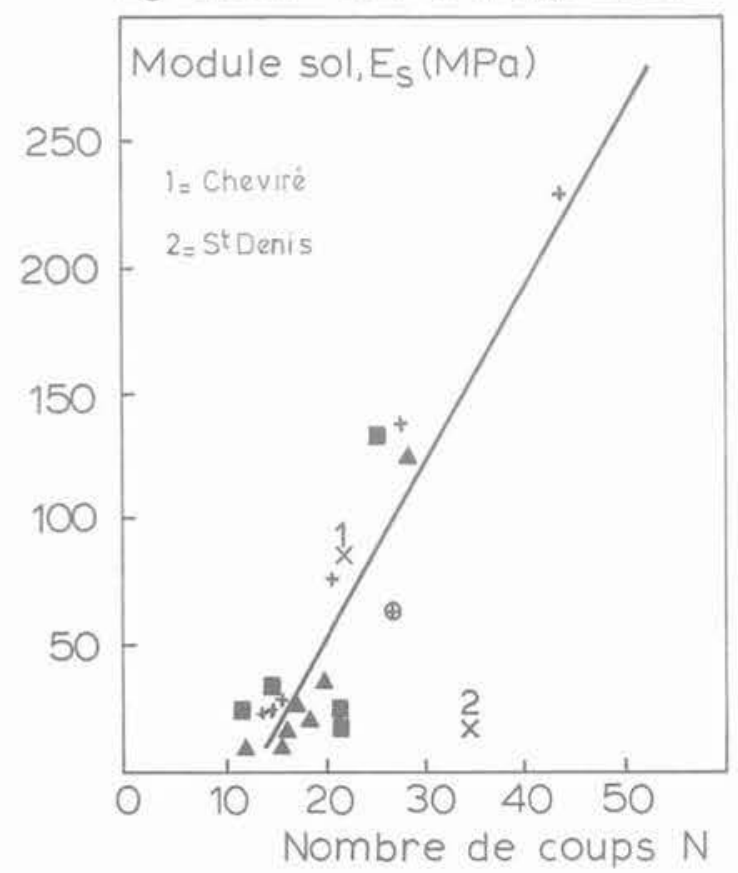

Pieux sans élargissement de la base $E_{S}=7,5 \mathrm{~N}-94,5(\mathrm{MPa}), r=0,98$

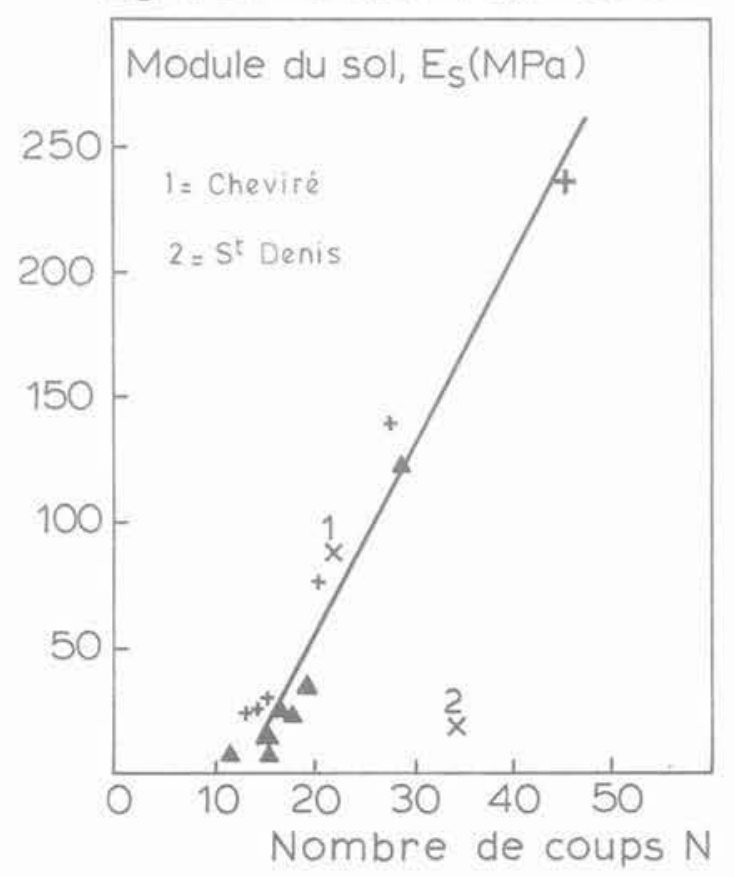

Fig. 3. - Corrélation entre le module du sol $E_{s}$ et $N$ (SPT) (CHRISTOULAS et PACHAKIS, 1987). Fig. 3. - Correlation between soil modulus $E_{S}$ and blow count $N$ (SPT).
(1) $E_{s}=21 q_{c}^{1,1}$
(2) $E_{s}=10 q_{c}$ $E_{\mathrm{S}} \leqslant 60 \mathrm{MPa}$
- pieux battus (Christoulas,1988) moulés
+ pieux forés

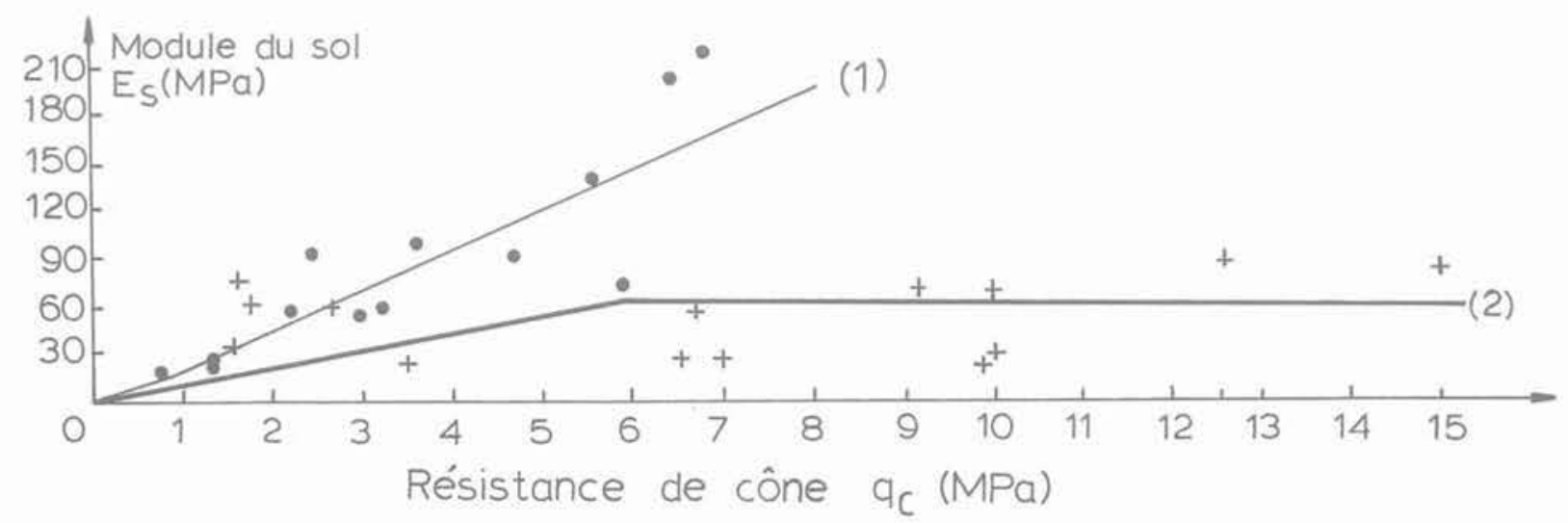

Fig. 4. - Corrélations entre le module du sol $E_{s}$ et la résitance au cône $q_{c}(C P T)$.

Fig. 4. - Correlations between soil modulus $E_{S}$ and cone resistance $q_{C}$ (CPT). 

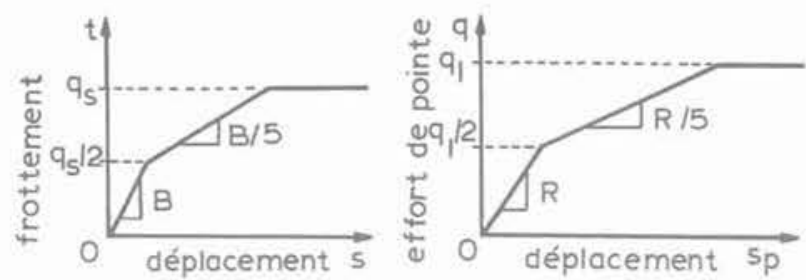

Fig. 5. - Lois de mobilisation du frottement latéral $(t, s)$ et de l'effort de pointe $\left(q, s_{p}\right)$ à partir des résultats de l'essai pressiométrique Ménard (PMT) (FRANK et ZHAO, 1982).

Fig. 5. - Side friction (t, s) and point pressure mobilization (q. $s_{p}$ ) laws from. Ménard pressuremeter tests (PMT) results.

une certaine adaptation. Les douze cas mentionnés plus bas sont extraits de cette étude (voir tableau 9).

\subsubsection{Le programme PIVER}

La méthode des fonctions de transfert de charge nécessite le recours à l'ordinateur, que ce soit tant à cause des non linéarités des courbes de mobilisation, qu'à cause de lhétérogénéité des couches. A cet effet le programme de calcul PIVER a été développé. II permet de prendre en compte les lois de mobilisation du frottement latéral et de l'effort de pointe les plus diverses, et notamment celles qui ont été proposées plus haut. Ses principes sont les suivants (FRANK, 1989).

L'équilibre d'une poutre, de module d'Young $E$ et de section d'aire $\mathrm{S}$, soumise à des efforts de compression ou de traction conduit à l'équation différentielle bien connue :

$$
\text { ES } \frac{d^{2} s}{d z^{2}}-P t\left(s-w_{s}\right)=0
$$

où : s est le déplacement longitudinal suivant $z$, et $\mathrm{Pt}$ la densité de force longitudinale répartie ( $\mathrm{P}$ est le périmètre du pieu au niveau considéré et $\mathrm{t}$ le frottement latéral unitaire, compté positivement lorsqu'il s'oppose au déplacement). $t\left(s-w_{s}\right)$ sont les courbes de mobilisation du frottement non linéaires telles que celles décrites plus haut, où l'on a introduit, de plus, un déplacement propre ou libre du sol (en l'absence de pieu) $w_{\mathrm{s}}$.

L'introduction du déplacement $w_{s}$ et le fait d'écrire que $t$ est une fonction non plus seulement du déplacement d'équilibre sol-pieu s, mais de la différence de déplacements $s-w_{s}$, permet d'effectuer des calculs de tassement de pieux dans des situations où se produit du frottement négatif. $w_{5}$ est le tassement de consolidation du sol à la profondeur considérée. Il varie, bien entendu, aussi avec le temps.

Si l'on se place sur un tronçon du système sol-pieu, pour lequel l'on peut considérer que :

- le pieu a une rigidité ES constante sur le tronçon :

- la courbe de mobilisation $\mathrm{t}\left(\mathrm{s}-\mathrm{w}_{\mathrm{s}}\right)$ est également unique ;

- et peut être linéarisée sous la forme :

$$
t=A+B \cdot\left(s-w_{s}\right) \text {, }
$$

où : $s-w_{\mathrm{s}}$ est la différence de déplacements au milieu de la couche ;

- la variation de $w_{\mathrm{s}}$ est linéaire en $\mathrm{z}$ : $\mathrm{w}_{\mathrm{s}}=\mathrm{a}_{0}+$ $a_{1}, z$, on obtient une équation différentielle à coefficients constants.

La solution analytique de cette équation dans chaque couche forme la base du programme de calcul automatique PIVER. L'originalité de PIVER réside dans le fait qu'il ne nécessite donc pas les approximations de la méthode des différences finies, habituellement utilisée dans les programmes similaires.

Dans le cas de $\mathrm{N}$ tronçons, les inconnues sont au nombre de $2 \mathrm{~N}$ (les 2 constantes d'intégration pour chaque tronçon). Les $2 \mathrm{~N}$ équations nécessaires sont fournies par:

- les 2 (N-1) conditions de continuité aux interfaces des tronçons : continuité du déplacement $\mathrm{s}$ et de l'effort axial $Q$;

- la condition de chargement en tête : charge axiale imposée ou déplacement axial imposé :

- et la condition en pointe : charge axiale imposée $\mathrm{Q}$ ou déplacement axial imposé $\mathrm{s}_{\mathrm{p}}$, ou charge axiale $\mathrm{Q}$ fonction du déplacement axial $\mathrm{s}_{\mathrm{p}}-\mathrm{w}_{\mathrm{sp}}$. La pression de pointe (charge unitaire) $\mathrm{q}$, est aussi linéarisée dans PIVER, sous la forme:

$$
\mathrm{q}=\mathrm{K}+\mathrm{R} \cdot\left(\mathrm{s}_{\mathrm{p}}-\mathrm{w}_{\mathrm{sp}}\right) \text {. }
$$

On aboutit alors à résoudre l'équation matricielle:

$$
|\mathrm{M}| \cdot|\mathrm{X}|=|\mathrm{Y}|
$$

où :

$|\mathrm{M}|$ est la matrice carrée $2 \mathrm{~N} \times 2 \mathrm{~N}$ contenant les valeurs en $\mathrm{z}$ des fonctions de $\mathrm{z}$;

(X) est le vecteur inconnu $2 \mathrm{~N}$ des constantes d'intégration ;

$\mathrm{Y} \mid$ le vecteur $2 \mathrm{~N}$ des valeurs imposées et des valeurs indépendantes de 2 .

L'utilisation de courbes non linéaires de mobilisation du frottement latéral ou de l'effort de pointe nécessite un processus de résolution itératif, pour trouver les valeurs finales des coefficients de linéarisation $A$, $\mathrm{B}, \mathrm{K}$ et $\mathrm{R}$. La convergence est obtenue lorsque les valeurs de $t$ (et de q) au milieu de chaque troncon (et à la pointe pour q) correspondent, à une précision fixée près, aux valeurs données par les courbes de mobilisation pour le même déplacement.

Chaque itération nécessite une nouvelle inversion de la matrice $|\mathrm{M}|$. Cette matrice est, en fait, une matrice bande, de largeur 5 qui s'inverse très rapidement sur les micro-ordinateurs actuels.

Le programme PIVER est d'un emploi très simple. Il permet de calculer la courbe charge-déplacement axial d'un pieu d'une manière rationnelle. Il s'agit, numériquement parlant, d'une méthode d'éléments discrets pour le sol et d'éléments à matrice de rigidité exacte pour le pieu, où la continuité des déplacements et des efforts axiaux est assurée. La seule discontinuité qui peut apparaître est, évidemment, la discontinuité des frottements latéraux aux interfaces. Pour le champ de frottements latéraux obtenu, PIVER donne la solution analytique rigoureuse des déplacements et des charges. 


\section{EXPLOITATION D'ESSAIS DE CHARGEMENT DE PIEUX FORÉS INSTRUMENTÉS}

Pour la présente étude on a évalué les différentes méthodes décrites dans le chapitre précédent, en analysant les résultats de 20 essais en vraie grandeur de pieux forés instrumentés, réalisés sur 16 sites différents par les Laboratoires de Ponts et Chaussées.

Les informations générales sur les essais et les sites traités sont données au tableau 8.

\subsection{Capacité portante}

Le tableau 8 compare les charges limites obtenues par les différentes méthodes aux charges observées dans la réalité.

Avant de commenter les résultats obtenus quelques remarques s'imposent. Tout d'abord, on ne dispose de résultats d'essais SPT (méthode 1) que dans deux cas et d'essais CPT (méthodes 2, 3 et 4) que dans 14 cas (avec refus avant d'atteindre la cote de la pointe du pieu dans 8 de ces cas).

En ce qui concerne le SPT, l'explication (en dehors de considérations sur les types de sols et leur aptitude à être testés) en est qu'il s'agit d'un essai très peu utilisé en France. Pour ce qui est du CPT, le manque d'information est généralement du au fait que les sols rencontrés ne se prêtaient pas, a priori, à ce type d'essai à cause de leur trop grande compacité. Le pressiomètre Ménard (PMT) (pour la méthode 5), par contre, a pu être mis en œuvre sur tous les sites.

Pour l'interprétation des essais de pieu, il faut mentionner que l'on a systématiquement cherché à appliquer toutes les méthodes, même lorsqu'elles ne sont pas recommandées pour certains sols. On a également dû procéder à certaines extrapolations : sous la cote de refus au CPT les profils de $\mathrm{q}_{\mathrm{c}}$ ont été supposés constants, le tableau 1 (méthode 3 ) a été utilisé pour des valeurs de $\mathrm{q}_{c}$ en dehors de la fourchette indiquée en supposant la proportionnalité de $q_{p}$, les tableaux 1 et 3 ont été utilisés pour les craies. lorsque l'on ne disposait pas de valeur de $c_{u}$ (tableau 4) on a utilisé la corrélation $c_{u}=q_{c} / 18$, etc.

La figure 6 regroupe l'ensemble de résultats du tableau 8 . On en tire les fourchettes et moyennes suivantes, pour le rapport charge limite calculée/observée :

- méthode 2 : 0,77 à 4,08, moyenne $=1,85$. Elle est souvent très optimiste ;

- méthode $3: 0,30$ à 0,95 , moyenne $=0,52$. Elle est souvent très pessimiste ;

- méthode 4: 0,64 à 1,50 , moyenne $=0,95$. Elle est modérément optimiste ou pessimiste, selon les cas ;
$1: \times$ SPT-CGS

$2: \bullet C P T-C G S$

3: $\square C P T-D I N$

4: $\triangle C P T-B G$

$5:+P M T$

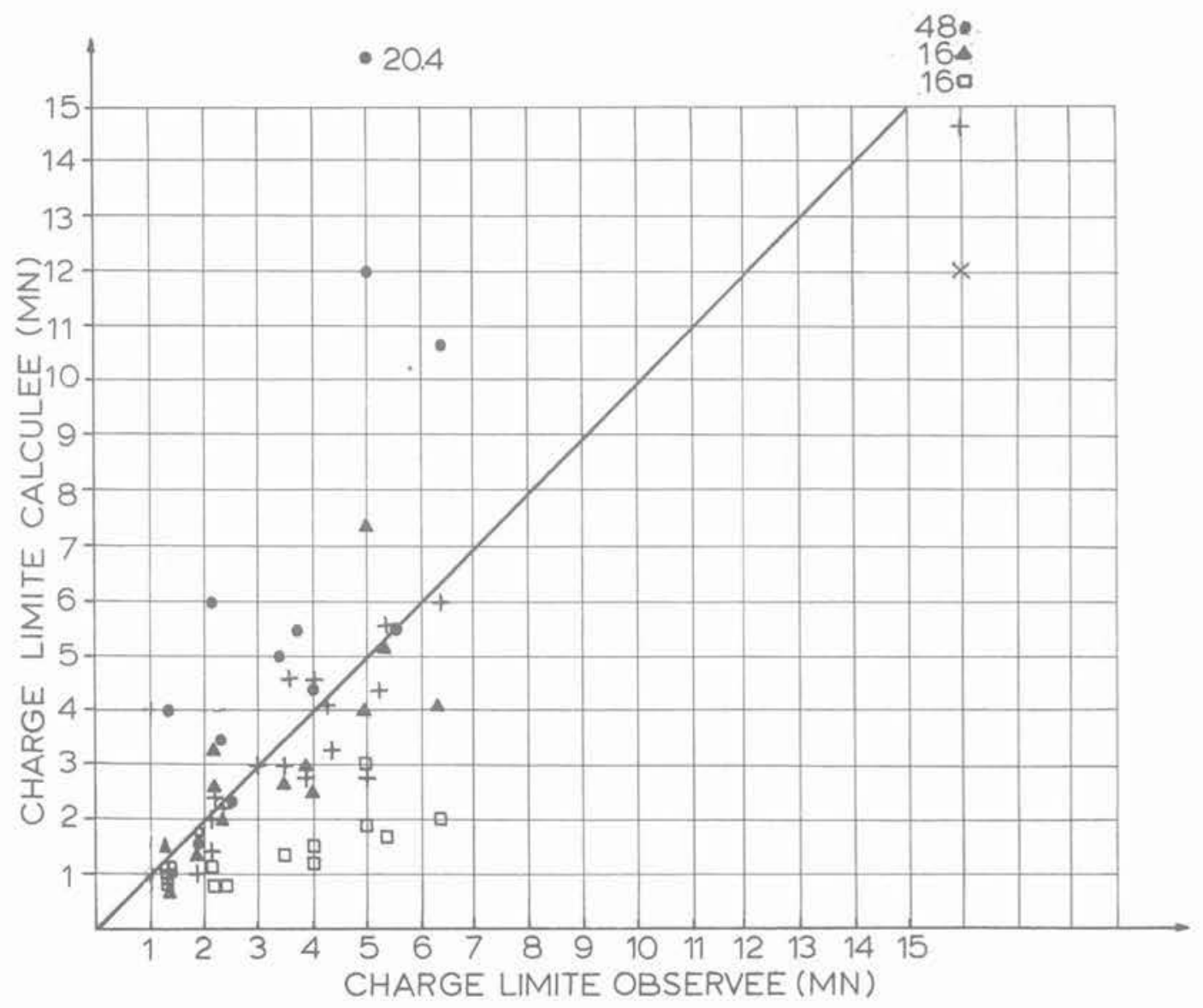

Fig. 6. - Comparaison entre charges limites observées et calculées.

Fig. 6. - Comparison between observed and predicted limit loads. 
Tableau 8. - Caractéristiques des essais de pieux et charges limites calculées par les 5 méthodes Table 8. - Piles tests data and limit loads determined by the 5 methods

\begin{tabular}{|c|c|c|c|c|c|c|c|c|c|c|c|}
\hline \multirow[b]{2}{*}{$N^{0}$} & \multirow[b]{2}{*}{ Site-Pieu } & \multirow[b]{2}{*}{ Sols } & \multirow{2}{*}{$\begin{array}{c}\text { Type } \\
\text { de pieu }\end{array}$} & \multirow{2}{*}{$\begin{array}{l}\text { Longueur } \\
D(m)\end{array}$} & \multirow{2}{*}{$\begin{array}{c}\text { Diamètre } \\
B(\mathrm{~m})\end{array}$} & \multirow{2}{*}{$\begin{array}{l}\text { Charge } \\
\text { limite } \\
\text { observée } \\
\text { MN }\end{array}$} & \multicolumn{5}{|c|}{ Charges limites calculées (MN) } \\
\hline & & & & & & & $\begin{array}{c}\text { (1) } \\
\text { SPT-CGS }\end{array}$ & \begin{tabular}{|c|} 
(2) \\
CPT-CGS
\end{tabular} & $\begin{array}{c}\text { (3) } \\
\text { CPT-DIN }\end{array}$ & $\begin{array}{c}\text { (4) } \\
\text { CPT-BG }\end{array}$ & $\begin{array}{c}\text { (5) } \\
\text { PMT }\end{array}$ \\
\hline 1 & Roubaix 1 & $\begin{array}{l}\text { Limon, } \\
\text { Argile }\end{array}$ & $\begin{array}{l}\text { foré tubé } \\
+ \text { simple }\end{array}$ & 20,2 & $\begin{array}{c}0,68 \\
\text { aे } 0,62\end{array}$ & 2,4 & & 2,3 & 0,8 & 2 & 2,3 \\
\hline 2 & Roubaix 2 & $\begin{array}{l}\text { Limon, } \\
\text { Argile }\end{array}$ & $\begin{array}{l}\text { foré tubé } \\
+ \text { simple }\end{array}$ & 27 & 0,88 & 5,4 & & 5,4 & 1,7 & 5,2 & 5,6 \\
\hline 3 & Lille & $\begin{array}{l}\text { Limon, } \\
\text { craie }\end{array}$ & $\begin{array}{l}\text { foré tubé } \\
+ \text { boue }\end{array}$ & 11,5 & $\begin{array}{c}0,6 \\
\text { à } \\
0,66\end{array}$ & $>4 *$ & & 4,3 & 1,2 & 2,6 & 4,5 \\
\hline 4 & Tourcoing & $\begin{array}{l}\text { Limon, } \\
\text { Argile }\end{array}$ & $\begin{array}{l}\text { foré tubé } \\
+ \text { boue }\end{array}$ & 25,3 & $\begin{array}{l}0,8 \\
\text { à } 1\end{array}$ & $6,4^{*}$ & & 10,7 & 2 & 4,1 & 6 \\
\hline 5 & Corbie & $\begin{array}{l}\text { Limon, } \\
\text { tourbe, } \\
\text { sable, craie }\end{array}$ & $\begin{array}{l}\text { foré tubé } \\
+ \text { boue }\end{array}$ & 12,5 & $\begin{array}{r}0,63 \\
\text { à } 0,9\end{array}$ & $2,2^{*}$ & & 3,3 & 0,8 & 3,3 & 2,5 \\
\hline 6 & Le Thiou 1 & $\begin{array}{l}\text { Marnes } \\
\text { compactes }\end{array}$ & $\begin{array}{l}\text { foré tubé } \\
+ \text { simple }\end{array}$ & 6 & 0,42 & 3,9 & & & & & 2,8 \\
\hline 7 & Le Thiou 2 & $\begin{array}{l}\text { Marnes } \\
\text { compactes }\end{array}$ & $\begin{array}{l}\text { foré tubé } \\
+ \text { simple }\end{array}$ & 6 & 0,56 & $>5,2^{*}$ & & & & & 4,3 \\
\hline 8 & Dunkerque & Sables & $\begin{array}{l}\text { foré smple } \\
+ \text { boue }\end{array}$ & 7,2 & $\begin{array}{c}0,43 \\
\text { à } 0,53\end{array}$ & 1,4 & & 4 & 1 & 1,7 & 0,8 \\
\hline 9 & Vendenheim 1 & $\begin{array}{l}\text { Sable, } \\
\text { Limon grave }\end{array}$ & $\begin{array}{l}\text { foré tubé } \\
+ \text { eau }\end{array}$ & 9,3 & 0,52 & $>3,8^{*}$ & & 5,5 & 1,5 & 3 & 2,7 \\
\hline 10 & Vendenheim 2 & $\begin{array}{l}\text { Sable, } \\
\text { limon grave }\end{array}$ & $\begin{array}{l}\text { foré tubé } \\
+ \text { eau }\end{array}$ & 7,9 & 0,52 & $>3,4^{*}$ & & 5 & 1.3 & 2,6 & 2,9 \\
\hline 11 & Colombes & $\begin{array}{l}\text { Limon, } \\
\text { sable grave }\end{array}$ & $\begin{array}{l}\text { tarière creu- } \\
\text { se Starsol }\end{array}$ & 7,3 & 0,98 & $>5 *$ & & 20,4 & 3 & 7,3 & 2,8 \\
\hline 12 & Merville & $\begin{array}{l}\text { Limon, } \\
\text { Argile }\end{array}$ & $\begin{array}{l}\text { tarière } \\
\text { creuse }\end{array}$ & 12 & 0,50 & 1,3 & & 1 & 0,9 & 0,9 & 1 \\
\hline 13 & $\begin{array}{l}\text { Boulogne- } \\
\text { Billancourt } 1\end{array}$ & Sable craie & $\begin{array}{l}\text { foré tubé } \\
+ \text { eau }\end{array}$ & 13 & 0,56 & 3 & & & & & 3 \\
\hline 14 & Cheviré & Sable & $\begin{array}{l}\text { foré tubé } \\
+ \text { boue }\end{array}$ & 30.1 & 1,15 & $16^{*}$ & 12 & 48 & 16 & 16 & 14,6 \\
\hline 15 & $\begin{array}{l}\text { Clermont- } \\
\text { Ferrand } 2\end{array}$ & $\begin{array}{l}\text { Limon, } \\
\text { sable }\end{array}$ & $\begin{array}{l}\text { tarière creu- } \\
\text { se Starsol }\end{array}$ & 10,35 & 0,82 & 1,9 & & 1,5 & 1,8 & 1,4 & 1 \\
\hline 16 & Toulouse & $\begin{array}{l}\text { Limon, } \\
\text { Argile } \\
\text { Marne argil. }\end{array}$ & $\begin{array}{l}\text { tarière creu- } \\
\text { se Starsol }\end{array}$ & 11,3 & $\begin{array}{l}0,55 \\
\text { à } 0,63\end{array}$ & 4,3 & & & & & 3,2 \\
\hline 17 & Saint-Denis & $\begin{array}{l}\text { Sable cal- } \\
\text { caire altéré }\end{array}$ & $\begin{array}{l}\text { foré tubé } \\
+ \text { simple }\end{array}$ & 9,8 & $\begin{array}{l}0,52 \\
\text { à } 0,60\end{array}$ & 2,1 & 1,4 & 6 & 1,2 & 2,6 & 2 \\
\hline 18 & Limay & $\begin{array}{l}\text { Alluvions } \\
\text { craie }\end{array}$ & $\begin{array}{l}\text { foré tubé } \\
+ \text { boue }\end{array}$ & 19,25 & $\begin{array}{c}0,63 \\
\text { a } 0,69\end{array}$ & 5 & & 12 & 1,9 & 4 & 3 \\
\hline 19 & Créteil A & $\begin{array}{l}\text { Remblai } \\
\text { marno- } \\
\text { calcaire }\end{array}$ & $\begin{array}{l}\text { foré tubé } \\
+ \text { boue }\end{array}$ & 16,5 & $\begin{array}{c}0,68 \\
\text { à } 0,76\end{array}$ & 3,6 & & & & & 4,6 \\
\hline 20 & Créteil B & $\begin{array}{l}\text { Remblai } \\
\text { marno- } \\
\text { calcaire }\end{array}$ & $\begin{array}{l}\text { foré tubé } \\
+ \text { boue }\end{array}$ & 16 & $\begin{array}{c}0,68 \\
\text { à } 0,76\end{array}$ & 4,2 & & & & & 4,1 \\
\hline
\end{tabular}

- Charge limite non atteinte. II s'agit d'une charge limite estimée. 
- méthode $5: 0.53$ à 1,28 , movenne $=0.86$. Même appréciation que pour la méthode 4.

Il peut paraitre normal que les méthodes 4 et 5 , mises au point à partir de la banque de données des Laboratoires des Ponts et Chaussées soient, pour diverses raisons, les plus fiables. Il faut signaler cependant, d'une part, que sur les 20 essais de pieux utilisés (voir tableau 8), seuls 8 faisaient partie de la banque disponible lors de la synthèse de BUSTAMANTE et GIANESELLI (1981), qui a servi de base à ces deux méthodes, et, d'autre part, qu'en ce qui concerne les 14 pieux pour lesquels la méthode CPT a été appliquée ici, seuls 3 avaient été utilisés en 1981. On notera de plus que les études récentes de BRIAUD et TUCKER (1988) et de ROBERTSON et al. (1988) ont montré, sur la base de données d'essais de pieux complètement indépendantes, tout le bien-fondé de la méthode CPT du LCPC.

\subsection{Tassement}

Tous les essais qui figurent au tableau 8 ont été analysés pour estimer les modules du sol équivalents $E_{s}$. en fonction du module pressiométrique $\mathrm{E}_{\mathrm{M}}$.
La procédure recommandée par POULOS et DAVIS (1980) a été utilisée, moyennant certaines hypothèses. On a estimé que le pieu était résistant en pointe lorsque le module pressiométrique en pointe était au moins égal au double du module pressiométrique moyen du fût. Le module pressiométrique moyen du fût est la moyenne arithmétique le long du fút, tandis que le module en pointe est la moyenne arithmétique de $D-1,5 B_{m}$ à $D+3 B_{m}, B_{m}$ étant le diamètre moyen du pieu.

La figure 7 donne pour tous les pieux le module du sol observé $E_{\mathrm{s}}$, déterminé par calcul à l'envers pour la charge nominale (charge admissible sous combinaisons quasi-permanentes) :

$$
\mathrm{Q}_{\mathrm{N}}=\mathrm{Q}_{\mathrm{C}} / 1,4
$$

où : $Q_{C}$ est la charge de fluage déterminée lors de l'essai.

Sur la figure 7 sont reportées les corrélations approximatives proposées entre le module du sol $E_{5}$ et le module pressiométrique moyen $\mathrm{E}_{\mathrm{M}}$ (approximations bilinéaires) :

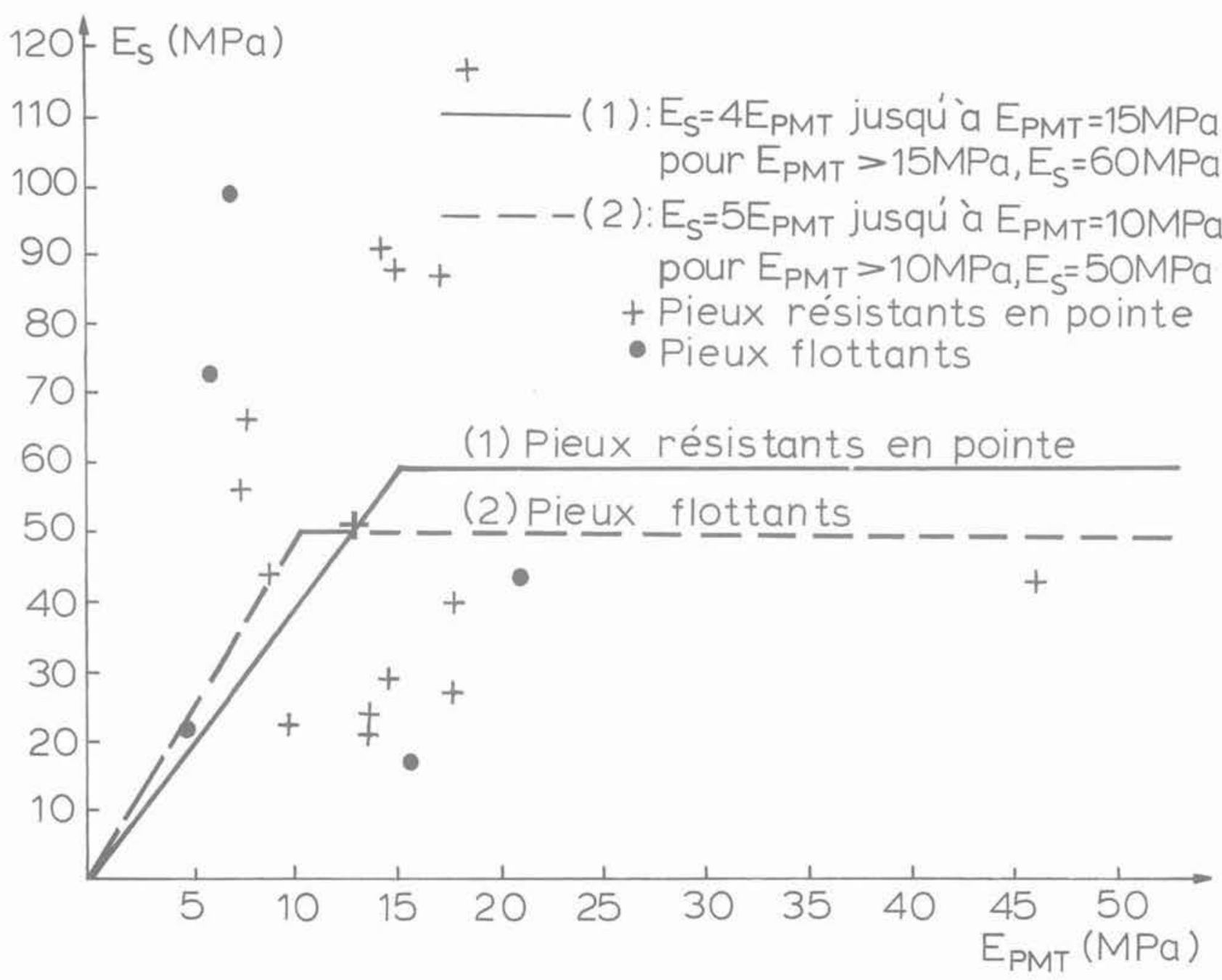

Fig. 7. - Corrélation entre le module du sol $E_{S}$ et le module pressiométrique $E_{P M T}$.

Fig. 7. - Correlation between soil modulus $E_{S}$ and pressuremeter modulus EPMT 
- pieux résistants en pointe :

et $\begin{aligned} E_{s} & =4 \mathrm{E}_{\mathrm{M}}, & \text { pour } \mathrm{E}_{\mathrm{M}}<15 \mathrm{MPa} \\ \mathrm{E}_{\mathrm{s}} & =60 \mathrm{MPa}, & \text { pour } \mathrm{E}_{\mathrm{M}}>15 \mathrm{MPa}\end{aligned}$

- pieux flottants :

$$
E_{s}=5 E_{M} \text {, pour } E_{M}<10 \mathrm{MPa}
$$$$
\text { et } E_{\mathrm{s}}=50 \mathrm{MPa} \text {, pour } \mathrm{E}_{\mathrm{M}}>10 \mathrm{MPa} \text {. }
$$

Les rapports des modules du sol calculés (en utilisant les corrélations proposées) aux modules du sol observés se situent entre 0,28 et 2,91 , ce qui montre bien la dispersion des résultats, ou l'approximation des corrélations... Les tassements sont évidemment dans les mêmes rapports. Cependant, ces corrélations peuvent constituer un outil d'évaluation de qualité équivalente à celles proposées par POULOS et DAVIS (1980) (voir fig. 2).

CHRISTOULAS (1988) a, quant à lui, proposé pour des pieux battus moulés la corrélation suivante avec la résistance au cône $\mathrm{q}_{c}$ (voir fig. 4);

$$
E_{s}=21 q_{c}^{1,1}
$$

L'analyse des 14 pieux pour lesquels on dispose de résultats CPT (et encore partiellement dans certains cas) semble indiquer, pour les pieux forés cette foisci, la corrélation (fig. 4) :

et

$$
\begin{array}{ll}
E_{5}=10 \mathrm{q}_{c}, & \text { pour } E_{M}<6 \mathrm{MPa} \\
E_{s}=60 \mathrm{Mpa}, & \text { pour } E_{M}>6 \mathrm{MPa}
\end{array}
$$

En ce qui concerne la méthode d'estimation du tassement à partir des fonctions de transfert de charge de FRANK et ZHAO (1982), elle a été appliquée, par BUSTAMANTE, FRANK et GIANESELLI (1989), à 12 pieux forés parmi les 20 étudiés ici. Le tableau 9 compare les tassements calculés aux tassements observés pour la même charge admissible que définie plus haut. Mis à part les deux cas de pieux forés dans des marnes argileuses déjà mentionnés, le rapport de ces tassements se situe entre 0,80 et 1,77 pour les autres sols et roches tendres, ce qui semble excellent, vu la précision habituellement recherchée pour estimer les tassements des pieux.

\section{CONCLUSIONS}

La confrontation des 5 méthodes de calcul de capacité portante des pieux à partir des résultats d'essais en place aux résultats de 20 essais de chargement de pieux forés réalisés en France, a permis de chiffrer la fiabilité de ces méthodes. Elle a, également, permis d'en évaluer la praticabilité. Certaines n'ont pu être appliquées que moyennant un certain nombre d'hypothèses ou d'extrapolation, forcément arbitraires et sans distinguer l'appareillage exact d'essai en place utilisé. Mais on mesure par là, en fait, le degré d'incertitude (certains diront le degré de marge...) laissé à l'ingénieur projeteur de fondations, qui ne peut pas choisir les sols du site du projet et n'est pas non plus toujours maître du type de pieu utilisé.

Même s'il serait exagéré de tirer des conclusions définitives sur ces exemples, finalement en nombre assez limité, on peut néanmoins mettre en avant tout l'intérêt de la méthode pressiométrique (SETRA et LCPC. 1985). Tout d'abord l'essai pressiométrique Ménard est réalisable dans tous types de sols et de roches tendres, du moins à terre, grâce à l'avant-trou. Les corrélations qui sous-tendent cette méthode et leur utilisation ne souffrent d'aucune incertitude majeure. Enfin, la dispersion obtenue est très raisonnable, lorsqu'on la compare aux autres méthodes, tant empiriques (à partir des essais en place, généralement), que théoriques (à partir des essais de laboratoire, généralement). C'est sans doute dû au fait qu'elle s'appuie sur des résultats d'essais en vraie grandeur de pieux réels et qu'elle distingue les différents modes d'exécution des pieux.

En ce qui concerne le calcul du tassement des pieux, on a pu proposer un ordre de grandeur pour le

\begin{tabular}{|c|c|c|c|c|}
\hline \multirow[t]{2}{*}{ Sols } & \multirow[t]{2}{*}{ Sites } & \multicolumn{2}{|c|}{$\begin{array}{l}\text { Tassements sous la charge nominale } \\
\qquad Q_{N}(\mathrm{~mm})\end{array}$} & \multirow[t]{2}{*}{$\begin{array}{l}\text { Rapport } \\
\text { (2) /(1) }\end{array}$} \\
\hline & & $\mathrm{s}_{\mathrm{N}}$ mesuré (1) & $s_{N}$ calculé $(2)$ & \\
\hline Argile & $\begin{array}{l}\text { Clermont-Ferrand } 2 \\
\text { Merville } \\
\text { Roubaix } 1 \\
\text { Roubaix } 2\end{array}$ & $\begin{array}{l}3,47 \\
1,69 \\
1,80 \\
3,74\end{array}$ & $\begin{array}{l}2,78 \\
1,69 \\
3,19 \\
4,70\end{array}$ & $\begin{array}{l}0,80 \\
1,00 \\
1,77 \\
1,26\end{array}$ \\
\hline Sable & Cheviré & 6,95 & 9,75 & 1,40 \\
\hline Craie & $\begin{array}{l}\text { Limay } \\
\text { Boulogne-Billancourt } 1\end{array}$ & $\begin{array}{l}4,20 \\
3,79\end{array}$ & $\begin{array}{l}7,05 \\
3,32\end{array}$ & $\begin{array}{l}1,68 \\
0,88\end{array}$ \\
\hline Marne argileuse & $\begin{array}{l}\text { Toulouse } \\
\text { Le Thiou } 2\end{array}$ & $\begin{array}{r}7,90 \\
22,31\end{array}$ & $\begin{array}{l}3,40 \\
4,11\end{array}$ & $\begin{array}{l}0,43 \\
0,18\end{array}$ \\
\hline Marno-calcaire & $\begin{array}{l}\text { Saint-Denis } \\
\text { Créteil A } \\
\text { Créteil B }\end{array}$ & $\begin{array}{l}2,73 \\
4,78 \\
4,74\end{array}$ & $\begin{array}{l}2,98 \\
5,37 \\
4,49\end{array}$ & $\begin{array}{l}1,09 \\
1,12 \\
0,95\end{array}$ \\
\hline
\end{tabular}

Tableau 9. - Comparaison entre tassements observés et tassements calculés (BUSTAMANTE, FRANK et GIANESELLI, 1989)

Table 9. - Comparison between observed and calculated settlements 
module d'Young à prendre en compte dans la méthode élastique de POULOS et DAVIS (1980), en fonction du module pressiométrique, ce qui devrait intéresser tant les praticiens français qu'étrangers, le pressiomètre étant le seul appareil d'essai en place permettant d'avoir un accès direct à la déformabilité du sol.

L'intérêt de la méthode $\mathrm{t}-\mathrm{z}$ de FRANK et $\mathrm{ZHAO}$ (1982), basée sur le module pressiométrique, et mise en ceuvre par le programme PIVER, est son aptitude à prendre directement en compte l'hétérogénéité et la non linéarité des lois d'interaction sol-pieu. Elle permet de calculer facilement toute la courbe chargetassement à partir des profils pressiométriques usuels. Elle devait se révéler être un complément intéressant à tout calcul de capacité portante par la méthode pressiométrique.

\section{REMERCIEMENTS}

Les auteurs tiennent à remercier tous les ingénieurs et techniciens du réseau des Laboratoires des Ponts et Chaussées, sans l'aide desquels les essais de pieux relatés ici n'auraient pas été réalisés.

En ce qui concerne les présentes interprétations, ils sont redevables des contributions de Luigi GIANESELLI, Polyxeni HARALAMBOPOULOU et Manolis DIMITROPOULOS, ces deux derniers y ayant consacré leur travail de fin d'études de l'Université Technique Nationale d'Athènes (1989).

Enfin, les auteurs sont reconnaissants envers la Commission mixte franco-hellénique (Ministère français des affaires étrangères et Secrétariat général hellénique à la recherche et à la technologie) qui a largement facilité l'accomplissement de cette étude en prenant en charge les missions d'échange nécessaires.

\section{BIBLIOGRAPHIE}

AFNOR (1990), Essai pressiomètrique Ménard. Norme PRP 94-110, 40 pages (à l'enquête au $1^{\text {et }}$ août 1990).

BRIAUD J.L., TUCKER L.M. (1988), Measured and predicted axial response of 98 piles. Jnl Geotech. Engng, ASCE, Vol. 114, $n^{\circ} 9$, September, p. 984-1001.

BOURGES F., FRANK R. (1989), Fondations Profondes. C 248-249, Techniques de l'Ingénieur, mai.

BUSTAMANTE M., GIANESELLI L. (1981), Prévision de la capacité portante des pieux isolés sous charge verticale. Règles pressiométriques et pénétrométriques. Bull. liaison Labo. P. et Ch., $\mathrm{n}^{\circ} 113$, mai-juin, p. 83-108.

BUSTAMANTE M., FRANK R., GIANESELLI L. (1989). Prévision de la courbe de chargement des fondations profondes isolées. Comptes rendus $12^{\mathrm{e}}$ Cong. Int. Méca. Sols et Tr. Fond., Rio de Janeiro, vol. 2, 15/6, pp. 1125-1126.

CASSAN M. (1966-1968), Le tassement des pieux ; synthèse des recherches récentes et essais com. paratifs. Sols Soils, $n^{\circ} 18-19$ (Première partie), 1966 , p. $43-58$ et $n^{\circ} 20$ (suite et fin), mars 1968 , p. $23-40$
CASSAN M. (1968), Les essais in situ en mécanique des sols, 1 . réalisation et interprétation. Eyrolles.

CGS (1985), Canadian Foundation Engineering Manual, 2nd edition. Canadian Geotechnical Society, c/o Bitech Publishers Ltd., Vancouver, BC.

CHARALAMBOPOULOU P. (1989), Evaluation comparative des méthodes de calcul de la charge limite des pieux à partir d'essais in situ. Travail de Fin d'Etudes de l'Université Technique Nationale d'Athènes, en grec.

CHRISTOULAS S., PACHAKIS M. (1987), Pile settlement prediction based on SPT results. Deltio, Bull. Centre Rech. TP (KEDE), n० 3, juil.-sept., p.221-226

CHRISTOULAS S. (1988), Dimensionnement des pieux. Quelques expériences et recherches en Grèce. Bull. liaison Labo. P. et Ch., $n^{\circ} 154$, mars-avril, p.5-10.

DIMITROPOULOS E. (1989), Tassement des pieux à partir du module pressiométrique du sol. Travail de Fin d'Etudes de l'Université Technique Nationale d'Athène, en grec.

DIN 4014 (1977), Bored piles. Large bored piles, manufacture, design and permissible loading. Part 2 , preliminary standard.

DTU n ${ }^{\circ} 13.2$ (1983), Fondations Profondes, chapitre 11: Calcul des fondations profondes soumises à charge axiale. Commentaires au cahier des charges de juin 1978, septembre, cahier 1877 , CSTB.

FRANK R. (1989), Déplacement axial des pieux dans les sols mous. Symposium franco-soviétique, Moscou (avril 1989). Laboratoire Central des Ponts et Chaussées, Rapport des Laboratoires, Série GT, $n^{\circ} 39$, novembre, pp. 105-114.

FRANK R., ZHAO S.R. (1982), Estimation par les paramètres pressiométriques de l'enfoncement sous charge axiale de pieux forés dans les sols fins, Bull. liaison Labo P. et Ch., $n^{\circ} 119$, maijuin, p. 17-24.

GAMBIN M. (1963), Calcul du tassement d'une fondation profonde en fonction des résultats pressiométriques. Sols Soils, $\mathrm{n}^{\circ} 7$, décembre, p. 11-31.

ISSMFE TC 16 (1989), Report of the ISSMFE Committee on Penetration Testing of Soils . TC 16 with Reference Test Procedures. CPT - SPT - DP -WST Swedish Geotechnical Institute, Information 7, june.

LCPC, SETRA (1985), Règles de justification des fondations sur pieux à partir des résultats des essais pressiométriques. Direction des Routes, octobre.

MEYERHOF G.G. (1976), Bearing capacity and settlement of pile foundations. Jnl of Geotech. Engng Div., ASCE, Vol. 102, n GT3, p. 195-228.

POULOS H.G., DAVIS E.H. (1980), Pile Founddation Analysis and Design. John Wiley and Sons.

ROBERTSON P.K., CAMPANELLA R.G., DAVIES M.P., SY A. (1988), Axial capacity of driven piles in deltaic soils using CPT.Proc. First Int. Symp. Penetration Testing (ISOPT-1), Orlando, Florida. 